\title{
An Analysis of Lower Leg Impactor Behavior by Physics Model"
}

\author{
Tsuyoshi YASUKI* \\ **Toyota Motor Corporation, \\ 1,Toyota-Cho, Toyota, Aichi, 471-8572, Japan \\ E-mail: yasuki@giga.tec.toyota.co.jp
}

\begin{abstract}
This paper describes development of a lumped mass-spring model of lower leg subsystem test of the European Enhanced Vehicle-safety Committee WG17 (here after referred as to the EEVC WG17) test method in order to provide relationship among the stiffness of the vehicle under the test, knee-bending angle and upper tibia acceleration of the lower leg subsystem impactor. The lower leg subsystem test and protection criteria are intended to protect against knee joint injuries and lower leg bone fracture. The knee-bending angle of the lower leg subsystem impactor was shown as a function of the stiffness of the vehicle under the test. The lumped mass-spring model results indicated that upper bumper stiffness forms a significant component of the knee-bending angle and lower bumper stiffness forms a significant component of upper tibia acceleration similar to upper bumper stiffness.
\end{abstract}

Key words: Lower Leg Impactor, Knee Joint Injury, Lumped Mass-Spring

1. Introduction

Pedestrians and cyclists form a significant proportion of all road user casualties in most countries. Lower leg subsystem test method was established to enhance pedestrian and cyclist protection ${ }^{(1)}$. After here, a lower leg impactor by the EEVC WG17 is referred as to "Impactor". Modifications to the vehicle to meet with the protection criteria by lower leg subsystem test of the EEVC WG17 test method will focus on the vehicle frontal shape and stiffness. Finite element analysis and physical tests are major tools to confirm that the modification will meet with the protection criteria of the lower leg subsystem test of the EEVC WG17 test method. The physical tests need a physical test prototype vehicle and the finite element analysis needs detailed three dimension geometry data of the vehicle, while the vehicle frontal shape and stiffness are not fixed at external styling stage ${ }^{(2)}$. If the tool that can investigate relationships between the stiffness of the vehicle under test and the knee-bending angle or upper tibia acceleration of Impactor is available at the external styling stage, the development of pedestrian and cyclist protector in vehicles will be enhanced.

The author established a lumped mass-spring model that can investigate the relationships between the stiffness of the vehicle under the test and the knee-bending angle and the upper tibia acceleration of Impactor. 


\section{Development of a lumped-mass spring model}

A lumped mass-spring model consists of masses and springs. A mass distribution of the lumped mass-spring model for Impactor was established by the investigation of the kinematics of Impactor obtained by a detailed finite element analysis result. A spring distribution of the lumped mass-spring model for Impactor was established by the investigation of the force transmissions between Impactor and the vehicle under the test obtained by the detailed finite element analysis result.

\subsection{Kinematics of Impactor}

The kinematics of Impactor was investigated by the detailed finite element analysis result. The author established finite element models of Impactor and the vehicle under the test for crash analysis code "LS-DYNA" version 960. Impactor FE model consists of shell elements and solid elements with fully deformable material properties (Fig. 1). A boundary and initial conditions of the finite element analysis are same as the lower leg subsystem test of the EEVC WG17 test method.

The kinematics of Impactor was assumed to be in a plane motion to simplify the constructions of the lumped mass-spring model. After here, the lumped mass-spring model is referred to as "Model". Model is assumed to move within the central section of the vehicle under the test. The vehicle central sections of the finite element analysis result are shown in Fig. 2. The kinematics of Impactor in Fig. 2 are as follows: a tibia of Impactor collides to upper and lower bumper and a femur of Impactor starts rotating clockwise at 11 ms. The tibia of Impactor rebounds from lower bumper. Femur of Impactor keeps on rotating clockwise and contacts to the hood edge at $20 \mathrm{~ms}$

The kinematics of Impactor in Fig. 2 can be regarded as the motions of two rigid bodies connected by a rotational joint at the knee.

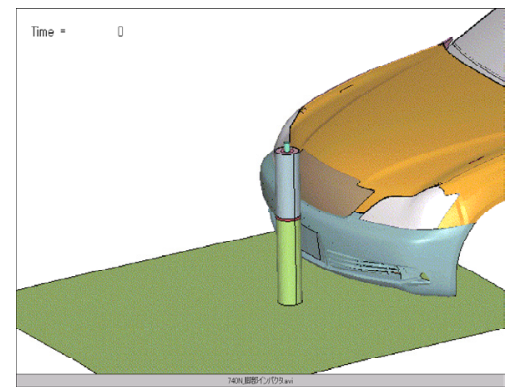

Figure 1. The detailed finite element model of Impactor and the vehicle under the test

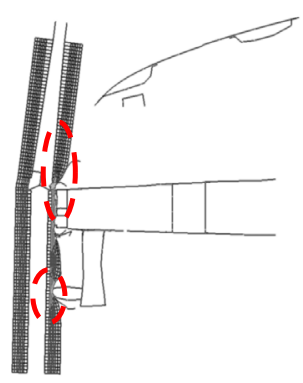

(a) $=11 \mathrm{~ms}$

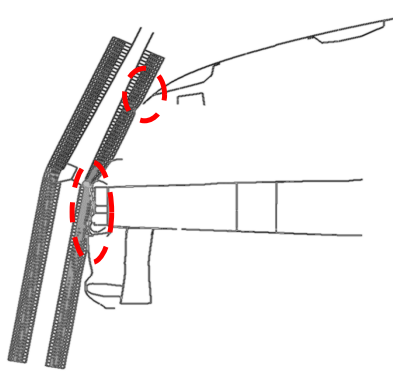

(b) $\mathrm{t}=20 \mathrm{~ms}$

Figure 2. Deformation of foam material of Impactor at central section 


\subsection{Force transmissions between Impactor and the vehicle}

By judging from deformation of foam materials of Impactor, force transmissions between Impactor and the vehicle under the test are as follows: the upper bumper and the lower bumper of the vehicle under the test contact to Impactor at $11 \mathrm{~ms}$ when the upper tibia acceleration indicates maximum value (Fig. 2 and 4). The upper bumper and the hood edge of the vehicle under the test contact to Impactor at $20 \mathrm{~ms}$ after the knee-bending angle indicates maximum values (Fig. 2 and 3 ).

The forces from the vehicle are transmitted to Impactor at three portions such as the lower bumper, the upper bumper, and the hood edge.

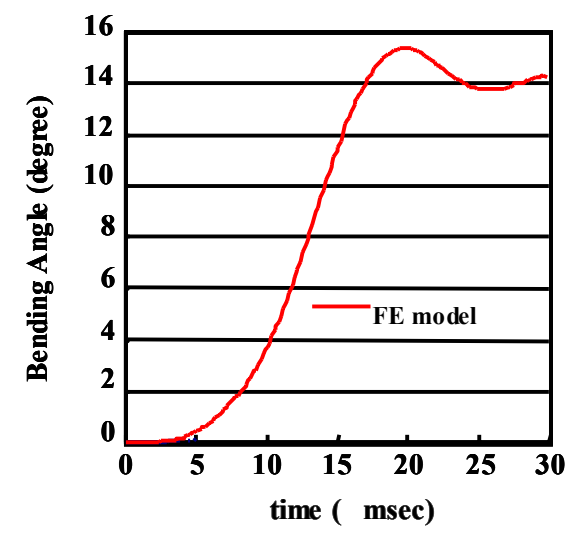

Figure 3. Knee-bending angle

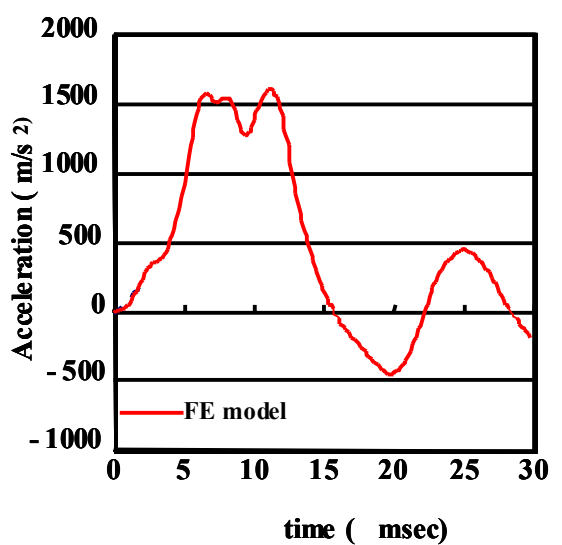

Figure 4. Upper tibia acceleration of Impactor

\subsection{Mass distribution of Model}

By taking account of the kinematics of Impactor described as previously, the femur and the tibia of Impactor of Model are assumed to be rigid bodies. The center of gravities, the masses, and the momentum inertias of Model are same as those of Impactor. A knee joint of Model is a mechanical rotational joint and the moment versus bending angle characteristic of the knee joint of Model is same as that of Impactor. Shearing stiffness of knee joint of Model is not available because the shearing deformation of the knee joint is much smaller than the displacement of the femur and the tibia.

\subsection{Spring distribution of Model}

By taking account of the forces transmitted from the vehicle under the test to Impactor described previously, forces transmitted from the vehicle under the test to Model are replaced by three non-linear scalar springs in $\mathrm{x}$ direction (Fig. 5). These spring characteristics include both stiffness foam material of Impactor and the vehicle under the test. They are extracted from finite element analysis results (Fig. 6 and 7). The displacements in the Fig. 5 are measured between rear end of front side rail of the vehicle 
under the test and the bone of Impactor. The displacement means summation of the deformation of the foam material of Impactor and the front of the vehicle. The forces in Fig. 5 are the contact forces between Impactor and the vehicle under the test.

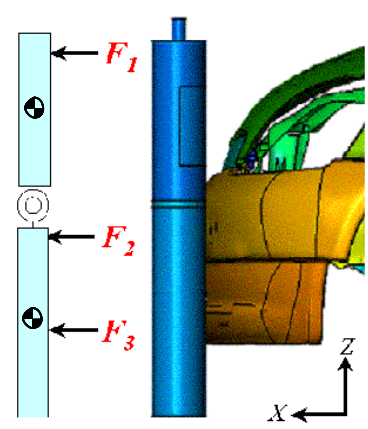

(a) Schematic view

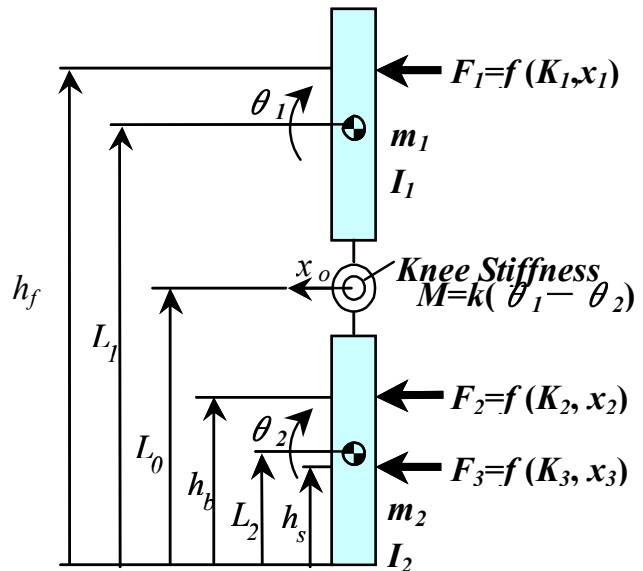

(b) Notation

Figure 5. Lumped mass-spring model

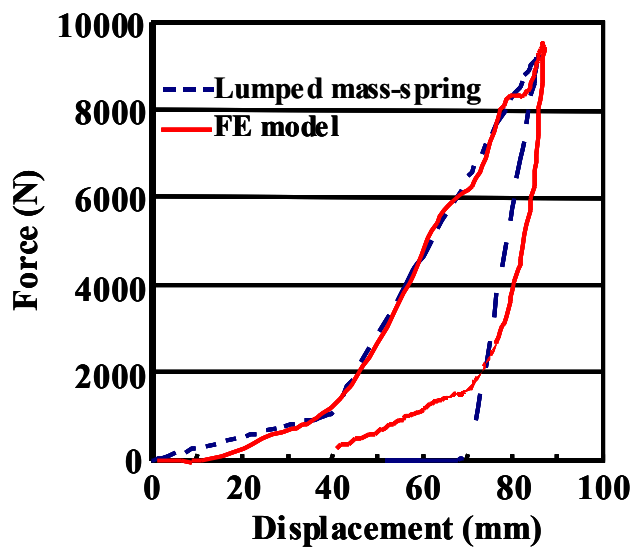

Figure 6. Force vs. displacement characteristics of K2

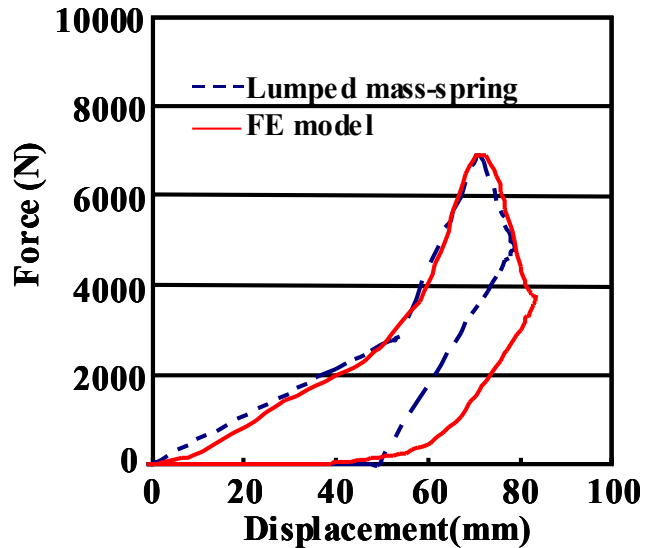

Figure 7. Force vs. displacement characteristics of K3 
3. Validation of the lumped mass spring model

The equations of motion for Model are described as follows by using the notations in Fig. 5:

$$
\begin{aligned}
& {\left[\begin{array}{ccc}
m_{1} L_{1}^{2}+I_{1} & 0 & m_{1}\left(L_{0}-L_{1}\right) \\
0 & m_{2} L_{2}^{2}+I_{2} & m_{2}\left(L_{2}-L_{0}\right) \\
-m_{1} L_{1} & m_{2} L_{2} & m_{1}+m_{2}
\end{array}\right]\left\{\begin{array}{l}
\ddot{\theta}_{1} \\
\ddot{\theta}_{2} \\
x_{0}
\end{array}\right\}+\left[\begin{array}{ccc}
k & -k & 0 \\
-k & k & 0 \\
0 & 0 & 0
\end{array}\right]\left\{\begin{array}{l}
\theta_{1} \\
\theta_{2} \\
x_{0}
\end{array}\right\}} \\
& =\left[\begin{array}{ccc}
L_{0}-h_{f} & 0 & 0 \\
0 & L_{0}-h_{b} & L_{0}-h_{s} \\
1 & 1 & 1
\end{array}\right]\left\{\begin{array}{l}
F_{1} \\
F_{2} \\
F_{3}
\end{array}\right\}
\end{aligned}
$$

Knee-bending angle of Model is described as follow by using the notations in Fig. 5:

$$
\theta=\theta_{1}-\theta_{2}
$$

Upper tibia acceleration of Model is described as summation of lateral and rotational acceleration by using the notations in Fig. 5 where constant value 0.066 is ratio of rotational acceleration increasing lateral acceleration.

$$
\alpha=\ddot{x}_{o}+0.066 \cdot \ddot{\theta}_{2}
$$

Equation [1] is integrated in time domain by Runge-Kutta scheme under the initial and boundary conditions similar to the lower leg subsystem test of the EEVC WG17 test method. The knee-bending angle and the upper tibia acceleration of Model are compared with those of the finite element analysis results of Impactor. They show similar time histories as those by the finite element analysis results (Fig. 8 and 9).

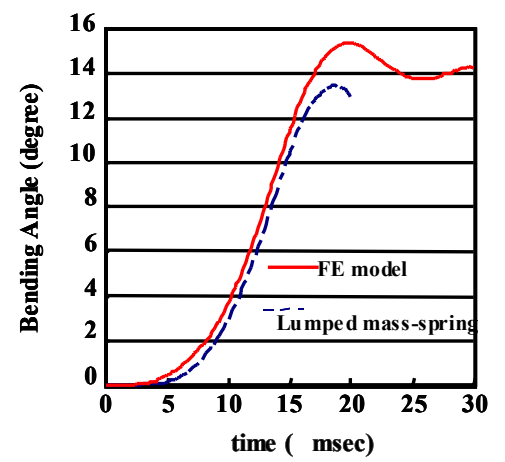

Figure 8. Comparison of knee-bending angle

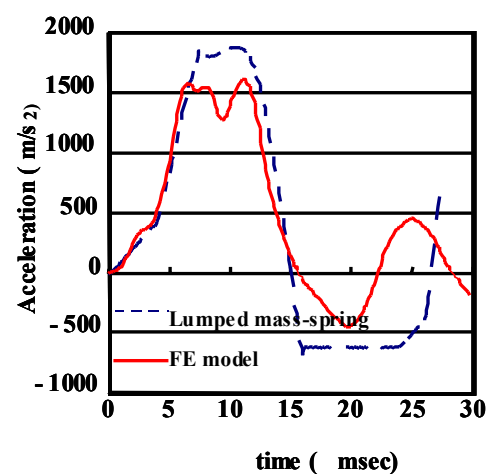

Figure 9. Comparison of upper tibia acceleration 
4. Knee bending angle and upper tibia acceleration as a function of contact forces

Equation [1] includes three independent variables $x 0, \theta 1$, and $\theta 2$. Substitution of Equation [3] to Equation [1] can eliminate the independent variable $x 0$ from Equation [1].

$$
\begin{aligned}
& {\left[\left(m_{1}+m_{2}\right)\left\{m_{1}\left(L_{1}-L_{0}\right)^{2}+I_{1}\right\}-m_{1}\left(L_{1}-L_{0}\right)\left\{m_{1}\left(L_{1}-L_{0}\right)-m_{2}\left(L_{0}-L_{2}\right)\right\}\right] \ddot{\theta}_{1}} \\
& -\left[\left(m_{1}+m_{2}\right)\left\{m_{2}\left(L_{0}-L_{2}\right)^{2}+I_{2}\right\}+m_{2}\left(L_{0}-L_{2}\right)\left\{m_{1}\left(L_{1}-L_{0}\right)+m_{2}\left(L_{0}-L_{2}\right)\right\}\right] \ddot{\theta}_{2} \\
& =\left\{-m_{1}\left(L_{1}-L_{0}\right)+m_{2}\left(L_{0}-L_{2}\right)-\left(m_{1}+m_{2}\right)\left(h_{f}-L_{1}\right)\right\} F_{1} \\
& +\left\{-m_{1}\left(L_{1}-L_{0}\right)-m_{2}\left(L_{0}-L_{2}\right)-\left(m_{1}+m_{2}\right)\left(h_{b}-L_{2}\right)\right\} F_{2} \\
& +\left\{-m_{1}\left(L_{1}-L_{0}\right)-m_{2}\left(L_{0}-L_{2}\right)-\left(m_{1}+m_{2}\right)\left(L_{2}-h_{s}\right)\right\} F_{3} \\
& -2\left(m_{1}+m_{2}\right) K\left(\theta_{1}-\theta_{2}\right)
\end{aligned}
$$

Equation [5] was derived by time integrating twice Equation [4] in order to provide the relationship between the knee-bending angle of Impactor and the stiffness of the vehicle under test explicitly.

$$
\begin{aligned}
& {\left[\left(m_{1}+m_{2}\right)\left\{m_{1}\left(L_{1}-L_{0}\right)^{2}+I_{1}\right\}-m_{1}\left(L_{1}-L_{0}\right)\left\{m_{1}\left(L_{1}-L_{0}\right)-m_{2}\left(L_{0}-L_{2}\right)\right\}\right] \theta_{1}} \\
& -\left[\left(m_{1}+m_{2}\right)\left\{m_{2}\left(L_{0}-L_{2}\right)^{2}+I_{2}\right\}+m_{2}\left(L_{0}-L_{2}\right)\left\{m_{1}\left(L_{1}-L_{0}\right)+m_{2}\left(L_{0}-L_{2}\right)\right\}\right] \theta_{2} \\
& =\left\{-m_{1}\left(L_{1}-L_{0}\right)+m_{2}\left(L_{0}-L_{2}\right)-\left(m_{1}+m_{2}\right)\left(h_{f}-L_{1}\right)\right\} \iint F_{1} d t^{2} \\
& +\left\{-m_{1}\left(L_{1}-L_{0}\right)-m_{2}\left(L_{0}-L_{2}\right)-\left(m_{1}+m_{2}\right)\left(h_{b}-L_{2}\right)\right\} \iint F_{2} d t^{2} \\
& +\left\{-m_{1}\left(L_{1}-L_{0}\right)-m_{2}\left(L_{0}-L_{2}\right)-\left(m_{1}+m_{2}\right)\left(L_{2}-h_{s}\right)\right\} \iint F_{3} d t^{2} \\
& \left.-2\left(m_{1}+m_{2}\right)\right] \int K\left(\theta_{1}-\theta_{2}\right) d t^{2}
\end{aligned}
$$

The tibia and the femur of Impactor have similar shapes and mass properties. Contact force F1 from the hood to the femur does not affect to the maximum knee-bending angle (Fig. 2 and 3). The knee joint of Model is the mechanical rotational joint and the moment versus bending angle characteristic of the knee joint of Model is almost constant, because the knee joint of Impactor is made of mild steel plate and the plastic bending deformation of the plate form whole resistance.

Assuming that the mass properties of the tibia of Impactor are same as those of the femur of Impactor (Equation 6, 7, and 8), ignoring effects of Contact force F1 to the knee-bending angle, and assuming that the bending angle characteristic of the knee joint of Model is constant (Equation 9), Equation 10 is obtained as an explicit description of $F 2, F 3$, and $M$.

The first and seconds terms of Equation 10 indicate the contributions of contact forces F2 and F3 to the knee-bending angle of Model simultaneously. The last term of Equation 8 indicate the contributions of the knee joint stiffness $\mathrm{M}$ to the knee-bending angle of Model.

$$
\begin{aligned}
& m 1=m 2 \\
& I 1=I_{2}=I \\
& L_{1}-L_{0}=L_{0}-L_{2}
\end{aligned}
$$




$$
K\left(\theta_{1}-\theta_{2}\right)=M
$$

where $\mathrm{M}$ is constant.

$$
I \theta=\left(h_{b}-L_{2}\right) \iint F_{2} d t^{2}-\left(L_{2}-h_{s}\right) \iint F_{3} d t^{2}-2 \iint M d t^{2}
$$

Contributions of the contact forces to the knee-bending angle by Equation 10 are shown in Fig. 10. Contact force $F 2$ between Impactor and the upper bumper of the vehicle under the test increases the knee-bending angle. Contact force $F 3$ between Impactor and the lower bumper decreases knee-bending angle. The knee joint stiffness $M$ decreases the knee-bending angle. Contact force $F 2$ between Impactor and the upper bumper of the vehicle under the test forms a significant proportion of the knee-bending angle of Model. More detailed investigation is necessary to determine the optimum value of contact force F2 and F3 for Impactor, by taking account of interactions between F2 and F3.

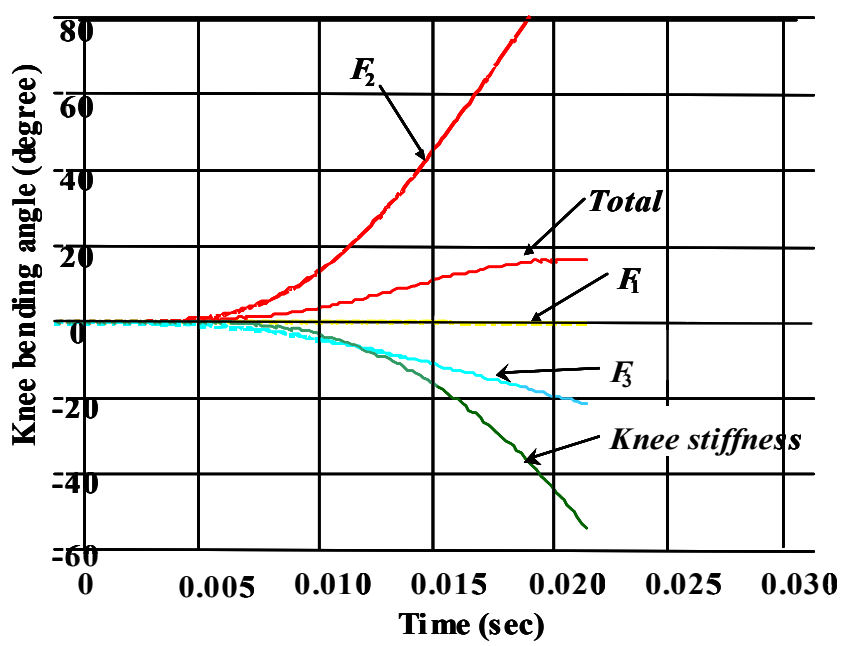

Figure 10. Contributions of the contact forces to the knee-bending angle

\section{Interaction of F2 and F3}

In order to determine the optimum value of contact force F2 and F3 for Impactor by taking account of interactions between F2 and F3, parametric studies by changing F2 and F3 were performed. These parametric studies are intended to change the frontal stiffness of the vehicle under the test. Scalar spring characteristics of K2 and K3 were modified as described in Fig. 11. Protection criteria are assumed that knee-bending angle is less than 15 degrees and upper tibia acceleration is less than $150 \mathrm{G}$. Because knee-bending angle and upper tibia acceleration of Model is exceeding the assumed protection criterion, the gradient of the curve of the force versus displacement where the frontal body deforms was reduced by $12.5 \%, 25 \%, 37.5 \%$ and $50 \%$ of the original gradient of K2 and K3. Five multiplied by five cases, (twenty-five cases) were performed by Model.

Knee-bending angle depends on relative stiffness K3 (Fig. 12). It is clearly shown that knee-bending angle of Model decreases as K3 increases. Upper tibia acceleration depends on both relative stiffness of $\mathrm{K} 2$ and $\mathrm{K} 3$. It is clearly shown that upper tibia acceleration of Model decreases as K2 and K3 decreases (Fig. 13). Increasing K3 is good for reducing knee-bending angle and is not good for upper tibia acceleration. Satisfying area of parameter $\mathrm{K} 2$ and $\mathrm{K} 3$ with the protection criteria of both knee-bending angle and upper tibia acceleration is shown in Fig. 14 as shaded area. 


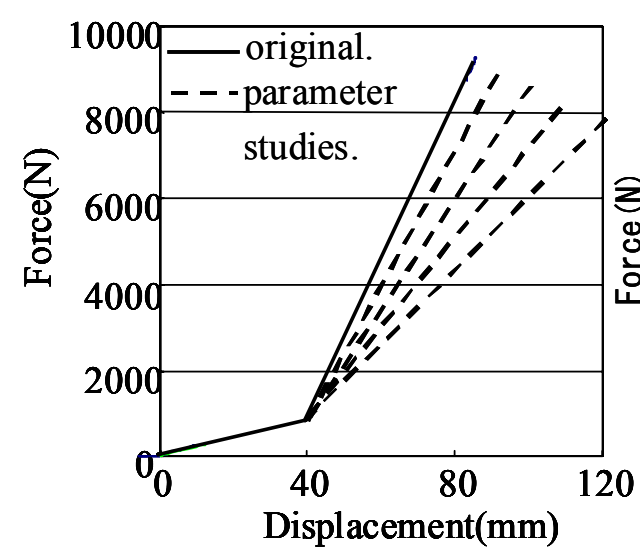

(a) $\mathrm{K} 2$

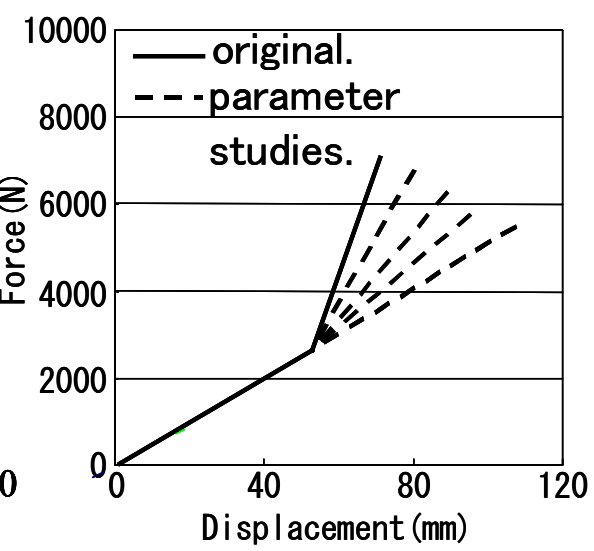

(b) $\mathrm{K} 3$

Figure 11. Force vs. displacement characteristics for K2 and K3 parameter studies

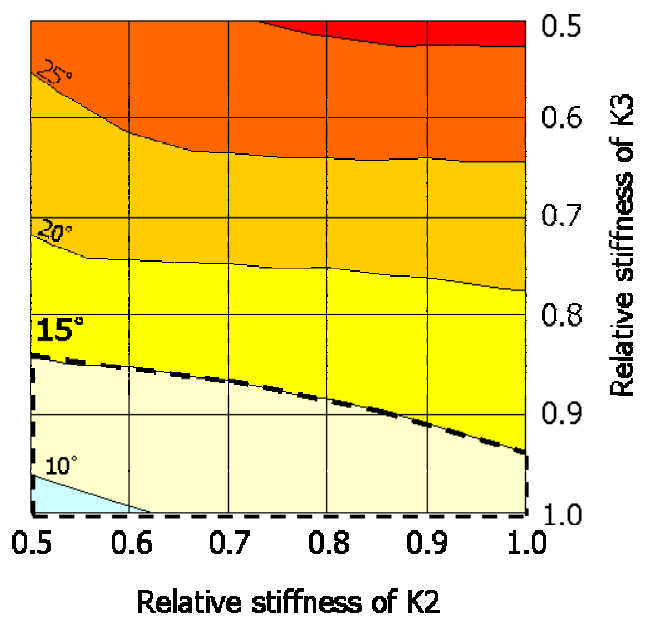

Figure 12. Interaction of $\mathrm{K} 2$ and $\mathrm{K} 3$ for knee-bending angle

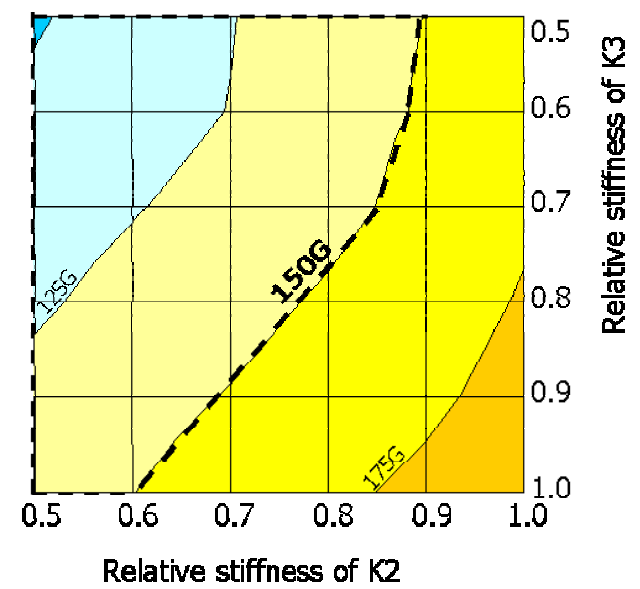

Figure 13. Interaction of K2 and K3 for upper tibia acceleration 


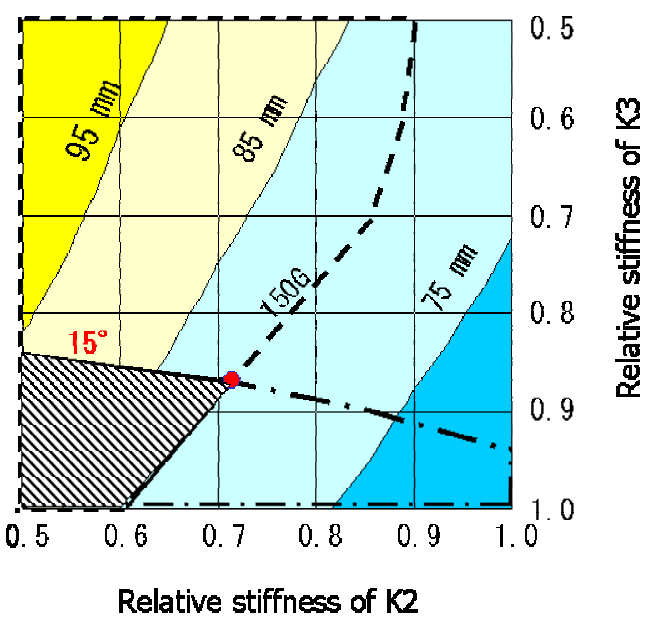

Figure 14. Interaction of $\mathrm{K} 2$ and $\mathrm{K} 3$ for displacement of $\mathrm{K} 2$

\section{Discussion}

According to parametric studies by Model of Impactor, lower bumper stiffness K3 forms significant component of knee-bending angle (Fig. 12). Contour map of knee-bending angle in Fig. 12 indicates that keeping certain level of stiffness of K3 is necessary to satisfy with the protection criteria of Impactor for the vehicle under the test. However, keeping certain level of lower bumper stiffness K3 may cause bone fracture at the central part of the tibia. It is hard to judge by using Model whether the central part of the tibia bone may fracture or not, because Impactor does not have an acceleration measurement device at central part of the tibia. As far as keeping certain level of stiffness of K3 is necessary to satisfy with the protection criteria of Impactor for the vehicle under the test, level of contact force F3 should be taken into account at a level that can keep protect against bone fracture at the central part of the tibia.

According to parametric studies by Model of Impactor, upper bumper stiffness K2 and lower bumper stiffness K3 form significant component of upper tibia acceleration of Impactor (Fig. 13). Upper tibia acceleration is measured at the tibia just below the knee joint. It is natural that contact force F2 correlates to bone fracture at tibia just below the knee joint, because contact force F2 is almost applied to same point as the bone fracture. However, it is not natural that contact force F3 also correlates to bone fracture at tibia just below the knee joint, although contact force F3 is applied to the central part of the tibia far from the knee joints. The reason why contact force F3 correlates to upper tibia acceleration of Impactor can be estimated that Impactor does not simulate bending and shearing deformation of the tibia. Rigid body rotation due to contact force F3 increases upper tibia acceleration.

\section{Conclusions}

A lumped mass-spring model for Impactor was established and validated with detailed finite element model results. The lumped mass-spring model result shows good correlation with the finite element model results.

Knee-bending angle was shown as a function of contacting forces by the lumped mass-spring model. Contact force by the upper bumper forms significant portion of the knee-bending angle.

Parametric studies by changing upper bumper stiffness and lower bumper stiffness are 
conducted by Model. Upper bumper stiffness forms a significant component of the knee-bending angle. Lower bumper stiffness forms a significant component of upper tibia acceleration similar to upper bumper stiffness.

\section{References}

(1) EURO-NCAP: EURO-NCAP's Pedestrian Impact Tests, version 4.0. Available at: http://www.euroncap.com. Access date 1st May 2007

(2) T Yasuki, 'A Survey on the Biofidelity of the Knee Bending Angle of the TRL Lower Leg Impactor', The 19th International Technical Conference on the Enhanced Safety of Vehicles (ESV), Washington D.C., NHTSA, 2005. 